\title{
Low temperature epitaxial silicon film growth using high vacuum electron-cyclotron-resonance plasma deposition
}

\author{
Scott J. DeBoer and Vikram L. Dalal \\ Department of Electrical and Computer Engineering, Iowa State University, Ames, Iowa 50011 \\ George Chumanov \\ Department of Chemistry, Iowa State University, Ames, Iowa 50011 \\ Randy Bartels \\ Department of Electrical Engineering, Oklahoma State University, Stillwater, Oklahoma 74078
}

(Received 28 November 1994; accepted for publication 21 February 1995)

\begin{abstract}
We report on the growth technique and electrical properties of epitaxial Si films grown at low temperatures using an electron-cyclotron-resonance plasma deposition technique. We have used standard high vacuum apparatus to grow high quality films at $450-525^{\circ} \mathrm{C}$. A critical step in achieving high quality films is an in situ hydrogen plasma cleaning of the wafer before growth. We have systematically studied the influence of ion bombardment during growth by biasing the substrate, and find that the films are crystalline for substrate bias voltages less negative than about $-15 \mathrm{~V}$, but become polycrystalline as the magnitude of the negative bias is increased. The crystallinity of the film was measured using Raman spectroscopy. The undoped films are $n$ type with carrier concentrations in the $10^{16}-10^{17} \mathrm{~cm}^{-3}$ range. The Hall mobilities measured for the films are comparable to values obtained in bulk Si crystals. We can achieve abrupt profiles in carrier concentrations between the heavy doped substrate and the epilayer, with no evidence of diffusion. (C) 1995 American Institute of Physics.
\end{abstract}

Low temperature epitaxial growth of silicon is of significant current interest due to its potential for making devices with small feature sizes. ${ }^{1}$ A low growth temperature (400$700{ }^{\circ} \mathrm{C}$ ) prevents the deleterious effects of autodoping from the substrate and lateral diffusion from contacts. To achieve such a low temperature growth, various techniques, such as ultrahigh vacuum chemical vapor deposition (UHV-CVD), ${ }^{1,2}$ very low pressure $\mathrm{CVD},{ }^{3}$ plasma enhanced $\mathrm{CVD},{ }^{4}$ and low pressure UHV electron-cyclotron-resonance (ECR) $\mathrm{CVD}^{5-8}$ have been used. The advantages of ECR-CVD are the low particulate production in the reactor, enhanced growth rate at low temperatures, and the ability to control plasma potentials, and hence ion bombardment of the substrate, during growth. In this paper, we report on a systematic study of conditions leading to the growth of very high quality Si films using a controlled, low pressure ECR plasma of silane and hydrogen at low temperatures $\left(450-525{ }^{\circ} \mathrm{C}\right)$.

The growth apparatus is similar to the one used in previous work by Mui et $a l^{8}{ }^{8}$ and Tae $e t ~ a l .{ }^{6}$ However, unlike these previous authors, we did not use a UHV system, but merely a standard high vacuum system equipped with O-rings. The system has previously been described in detail. ${ }^{9}$ The base pressure in the system is in the range of 5-9 $\times 10^{-8}$ Torr. These vacuum conditions require the growth rate to be maximized in order to reduce the incorporation of impurities as previously described by Comfort and Reif. ${ }^{3}$ Most of the current techniques for growing epitaxial silicon films below $600{ }^{\circ} \mathrm{C}$ require UHV conditions due to their extremely low growth rates $(<1 \AA / \mathrm{s}){ }^{2,5-8}$ In contrast, the enhanced surface mobility of radicals due to ion bombardment in the ECR plasma allows typical growth rates of more than $3.5 \AA / s$ to be achieved. The growth rate in this type of system is very sensitive to the process conditions, especially the pressure, and silane to hydrogen ratio. We used hydrogen as the plasma gas, with a typical flow rate of $30 \mathrm{sccm}$. Silane was introduced close to the substrate at a flow rate of 10 sccm, thus giving a dilution ratio of $\mathrm{H}_{2} / \mathrm{SiH}_{4}$ of $3: 1$. We found the $\mathrm{H}_{2} / \mathrm{SiH}_{4}$ ratio to be a very important parameter in determining the film quality. Further reduction of the dilution ratio leads to rough polycrystalline films while increasing the ratio leads to a reduction in the growth rate. We have deposited specular films at pressures between 6 and $50 \mathrm{mT}$ and at temperatures between 425 and $600{ }^{\circ} \mathrm{C}$. The upper limit to the growth temperature regime has previously been attributed to the rapid desorption of hydrogen, leaving the surface unprotected from contamination during growth. ${ }^{10}$ The chamber was equipped with a shutter, so that the system could be plasma cleaned before the growth of the epitaxial layer without depositing on the substrate. This same plasma cleaning was also found to be useful between the growth of doped and undoped layers, so as to minimize any cross contamination between multiple layers of a devices. ${ }^{11}$

We monitored the electron temperatures, plasma densities, and plasma potentials next to the substrate by using Langmuir probe measurements. The measurement procedure has been outlined previously. ${ }^{12}$ Typical results for the plasma parameters are shown in Fig. 1. It is clear that as the pressure is decreased there is an increase in both the electron temperature and the ion bombardment energy. The maximum observed in the plasma density in Fig. 1(b) is consistent with previous results. ${ }^{13}$ Our substrate can be biased with respect to the chamber walls during growth, allowing us to control the energy of the incident ions. We have studied the influence of this bias voltage on the structural and electrical properties of silicon films.

Before introducing the Si wafers into the system, they 


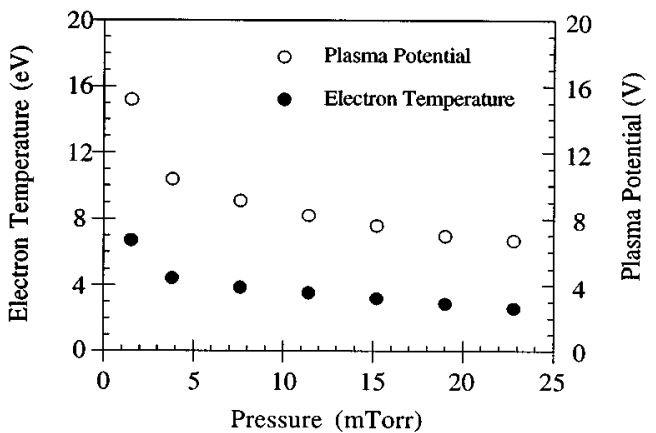

(a)

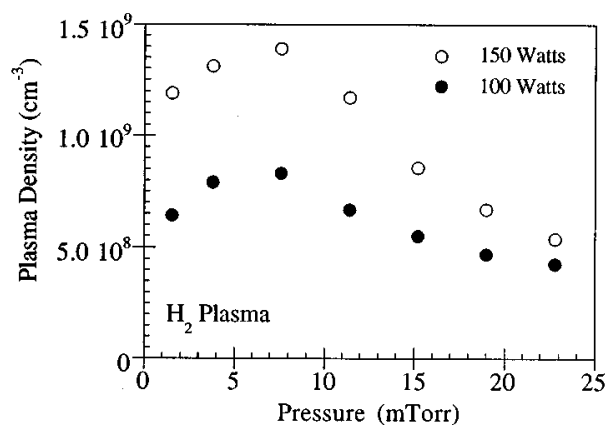

(b)

FIG. 1. Langmuir probe measurements showing the variation of important plasma parameters with pressure: (a) plasma potential and electron temperature and (b) plasma density.

were cleaned with a standard RCA process. Just prior to the introduction of the wafers into the chamber, they were dipped into a 50:1 $\mathrm{H}_{2} \mathrm{O}: \mathrm{HF}$ solution. To remove the thin oxide layer and to leave the wafer surface passivated with hydrogen. If this HF dip was not used, the films turned out to be polycrystalline with rough surfaces. This observation is consistent with the model proposed by Meyerson ${ }^{11}$ that $\mathrm{H}$ passivation is the critical step in achieving successful low temperature epitaxial growth of Si.

After introduction of the wafers into the reactor the wafers were etched using a hydrogen plasma from the ECR source. This hydrogen plasma etch was found to be important in achieving reproducible growth of high quality films. This fact is not surprising, since hydrogen plasmas produced by ECR sources have been shown to be effective gettering agents for surface contaminants such as $\mathrm{O}$ and $\mathrm{C} .{ }^{13}$ Since we are only using a high vacuum system, such contaminants are likely to be present, and the in situ hydrogen plasma etch effectively removes them from the surface and from the reactor walls. In contrast to previous results, ${ }^{6}$ our transmission electron microscope (TEM) images have shown that no surface roughening is present due to the plasma exposure. This may be a result of the lower ion energy, used in this work.

The films reported in this work were all grown with a substrate temperature of $525^{\circ} \mathrm{C}$ at a reactor pressure of 12 mT. The substrate was biased using a dc source, and this bias voltage was systematically varied for different runs. The thicknesses of the films in this work were 1.5-2.0 $\mu \mathrm{m}$. The crystallinity of the films was measured using Raman spectroscopy. In Fig. 2(a), we show the typical Raman spectra of

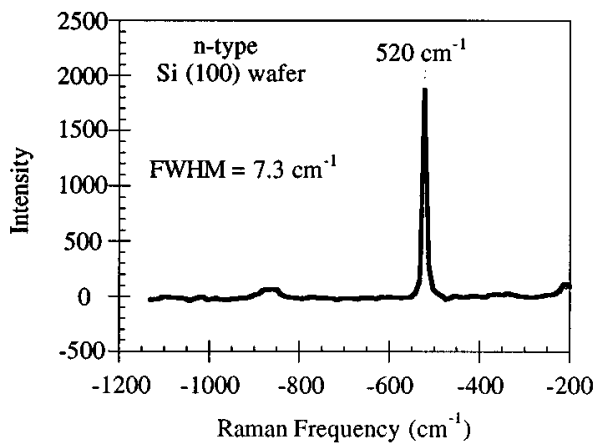

(a)

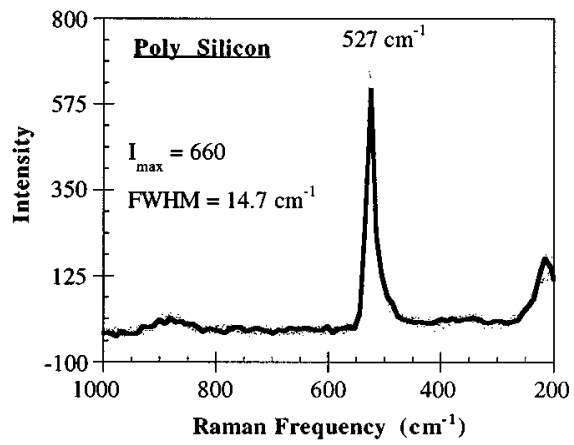

(b)

FIG. 2. Typical Raman spectra of (a) Si (100) wafer, (b) poly Si film. The intensity, peak position, and FWHM are all indications of the film quality.

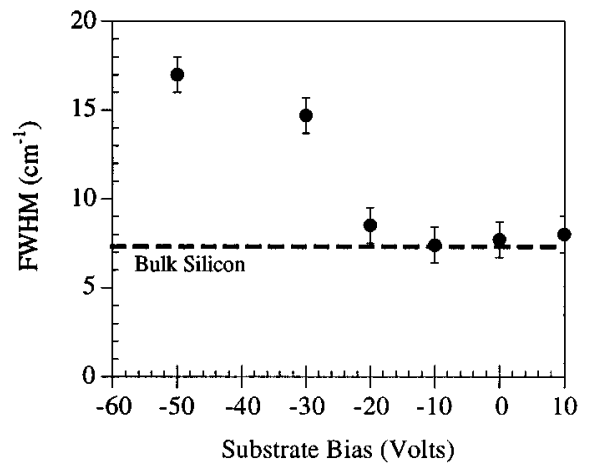

(a)

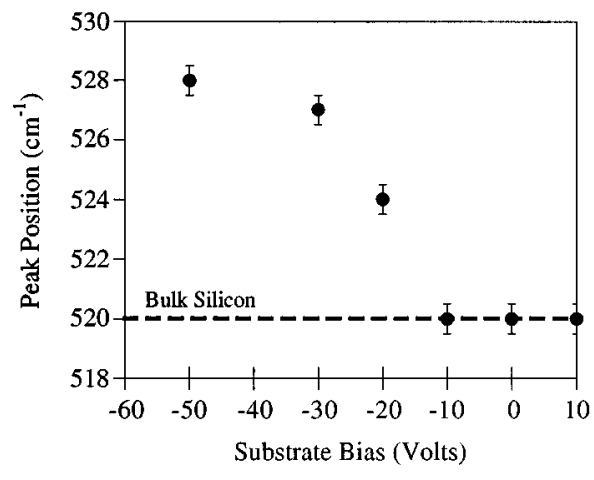

(b)

FIG. 3. Raman results for a systematic study of substrate bias effects in ECR-CVD grown films. The sharp transitions in both (a) the FWHM and (b) the peak position indicates the conditions under which growth changes from crystalline to polycrystalline. 


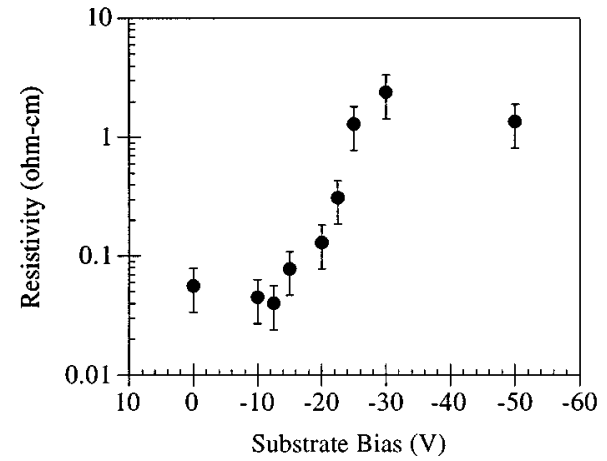

(a)

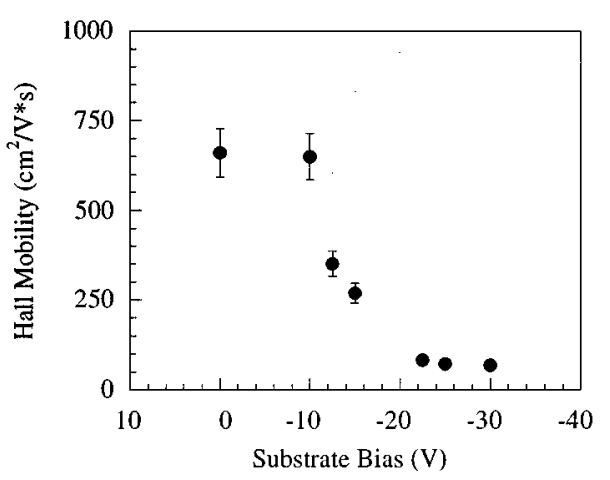

(b)

FIG. 4. Variation of (a) the resistivity and (b) Hall mobility of Si films as a function of substrate bias.

a Si (100) wafer, and in Fig. 2(b), the Raman signal for an ECR-grown polycrystalline Si film is shown. Note that for poly Si films, there is a shift in the peak position, and there is significant broadening of the peak, leading to an increase in full width at half maximum (FWHM). In Fig. 3, we show the influence of varying substrate bias voltage on both FWHM and peak position of the Raman signal. It is apparent from this figure that increasing the magnitude of the negative bias voltage leads to an abrupt change in the crystallinity of the film at about $-15 \mathrm{~V}$ bias voltage, or about $-25 \mathrm{~V}$ total voltage difference between the plasma sheath and the substrate. For greater bombardment energies, the films are polycrystalline, as indicated by the increasing FWHM and the shift in the peak position of the Raman signal. This result is consistent with the earlier result reported by Nagai et al. ${ }^{5}$

We measured Hall mobilities in the material. In Fig. 4, we show the resistivity and electron mobilities as a function of substrate bias voltage. All of the undoped films were $n$ type. For substrate biases between 0 and $-10 \mathrm{~V}$ the resistivities shown in Fig. 4(a) correspond to carrier concentrations of about $2 \times 10^{17} \mathrm{~cm}^{-3}$. Once again, we find that at about $-15 \mathrm{~V}$ substrate bias, there is an abrupt increase in resistivity of the film, and a corresponding reduction in Hall mobilities, confirming the interpretation of the Raman results. The Hall mobilities measured for our crystalline films correspond very

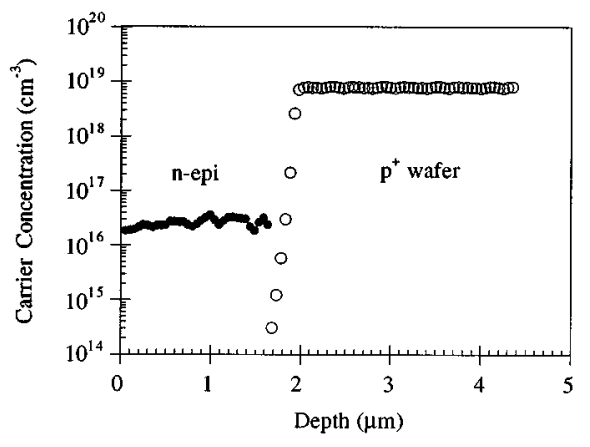

FIG. 5. Typical spreading resistance profile for an undoped epitaxial silicon film grown using ECR-CVD at $525^{\circ} \mathrm{C}$.

well to the bulk mobility value of $600 \mathrm{~cm}^{2} / \mathrm{V}$ s reported in the literature ${ }^{14}$ for $n$-type silicon with $N_{D}=2 \times 10^{17}$ $\mathrm{cm}^{-3}$.

In Fig. 5, we show the carrier concentration of an epitaxial film. The low doping concentration (a few $10^{16}$ $\mathrm{cm}^{-3}$ ), and the abrupt junction edge between the substrate and the epilayer are noteworthy features.

In summary, we report on the growth of high quality epitaxial Si films using ECR plasma deposition in a high vacuum reactor. By using plasma cleaning of the wafer before growth, we can remove the contaminants present in such reactors. We have systematically measured the effects of ion bombardment on the electronic and structural properties of the films, and find that ion bombardment voltages greater than about $25 \mathrm{~V}$ cause damage to the film, and result in polycrystalline films. The high vacuum ECR plasma deposition technique is shown to produce films with excellent electron mobilities and low donor densities, and also produces layers with abrupt interfaces. We can also achieve high growth rates of $3-5 \AA / s$.

This work was supported in part by a fellowship from the Catron foundation for Scott DeBoer, and in part by a grant from NREL. We thank R. Knox, M. Stoops, and M. Leonard for many interesting discussions.

${ }^{1}$ B. Meyerson, Appl. Phys. Lett. 48, 797 (1986).

${ }^{2}$ B. S. Meyerson, IBM J. Res. Dev. 34, 806 (1990).

${ }^{3}$ J. Comfort and R. Reif, J. Electrochem. Soc. 136, 2386 (1989).

${ }^{4}$ J. Comfort and R. Reif, J. Electrochem. Soc. 136, 2398 (1989).

${ }^{5}$ I. Nagai, T. Takahagi, A. Ishitani, H. Kutoda, and M. Yoshikawa, J. Appl. Phys. 64, 5183 (1988).

${ }^{6}$ H. S. Tae, S. Hwang, S. Park, E. Yoon, and K. Whang, Appl. Phys. Lett. 64, 1021 (1994)

${ }^{7}$ H. Yamada and Y. Torii, J. Appl. Phys. 64, 702 (1988).

${ }^{8}$ D. S. Mui, S. F. Fang, and H. Morkoç, Appl. Phys. Lett. 59, 1887 (1991).

${ }^{9}$ S. J. DeBoer and V. L. Dalal, First World Conference on Photovoltaic Energy Conversion (to be published).

${ }^{10}$ B. Meyerson, F. Himpsel, and K. Uram, Appl. Phys. Lett. 57, 1034 (1990).

${ }^{11}$ H. Ugur, S. Varol, S. Ugur, and I. Karabay, J. Non-Cryst. Solids 137\&138, 761 (1991).

${ }^{12}$ R. D. Knox, V. L. Dalal, and O. A. Popov, J. Vac. Sci. Technol. A 9, 474 (1991).

${ }^{13}$ M. Ishii, K. Nakashima, I. Tajima, and M. Yamamoto, Appl. Phys. Lett. 58, 1378 (1991).

${ }^{14}$ G. Baccarani and P. Ostoja, Solid State Electron. 18, 579 (1975). 